\title{
Influence of the Track Forecast of Typhoon Prapiroon on the Heavy Rainfall in Western Japan in July 2018
}

\author{
Takeshi Enomoto \\ Disaster Prevention Research Institute, Kyoto University, Uji, Kyoto, Japan
}

\begin{abstract}
The predictability of the Heavy Rain Event in July 2018 is examined by forecast experiments with an operational global atmospheric forecast model. Experiments from different initial times show that the overall rainfall distribution at the peak on 6 July can be predicted from 12 UTC, June 30, and later. In the successful forecasts, the track error of Typhoon Prapiroon against the best track is small. In the experiments with longer lead times, by contrast, the Baiu frontal zone has a northwared bias with less precipitation, and Prapiroon hardly develop and migrates westward. Poor track forecasts seem to be related to the limited vertical development of the vortex. Near surface equivalent potential tempeature and Q-vector analysis show that Prapiroon act to intensify the Baiu frontal zone. In conclusion, the correct track forecast is essential for Baiu frontogenesis and the formation of heavy precipitation in western Japan.

(Citation: Enomoto, T., 2019: Influence of the track forecast of Typhoon Prapiroon on the heavy rainfall in western Japan in July 2018. SOLA, 15A, 66-71, doi:10.2151/sola.15A-012.)
\end{abstract}

\section{Introduction}

During the Heavy Rain Event of July 2018 (HRE1807 hereafter), landslides and floods occurred mainly in western Japan. Typhoon Prapiroon (named after the god of rain in Thai) followed by an intensified Baiu frontal zone (BFZ) caused record-breaking precipitation.

The Japan Meteorological Agency (JMA) listed a few factors that may have contributed to the heavy rainfall based on their analysis and discussions with experts as follows (Shimpo et al. 2019):

1. Continuous convergence of two streams with large moisture in western Japan (Sekizawa et al. 2019; Takemura et al. 2019)

2. Intensified ascent due to the stagnation and intensification of the BFZ

3. Formation of local line-shaped precipitation bands (Tsuguti et al. 2018; Takemi and Unuma 2019)

However, the mechanisms and predictability of the intensification of the BFZ are not clear.

Kotsuki et al. (2019) conducted 100-member ensemble forecast experiments to investigate the predictability of HRE1807. They independently noted the improvement in precipitation as the forecast track of Prapiroon becomes closer to the observed track. They concluded that HRE1807 can be predicted three days in advance with their system but with slightly northward bias. They speculated that the bias is due to the coarse model resolution of $112 \mathrm{~km}$. Matsunobu and Matsueda (2019) compared operational ensemble forecasts and conducted ensemble forecast experiments with a somewhat finer horizontal resolution of approximately $78 \mathrm{~km}$, which is still insufficient to resolve a tropical cyclone.

Moteki (2019) showed that Prapiroon drew cold air from the Okhotsk anticyclone that intensified the temperature and pressure gradient. Our forecast experiments would complement Moteki's analysis to answer a question whether the BFZ might have still

Corresponding author: Takeshi Enomoto, Disaster Prevention Research Institute, Kyoto University, Gokasho, Uji, Kyoto 611-0011 Japan. E-mail: enomoto.takeshi.3n@kyoto-u.ac.jp. intensified if it were not for the typhoon.

The purposes of the present study are to clarify the predictability of the heavy rainfall, to explore the intensification mechanisms of the BFZ, and to examine the influence of the typhoon on the intensification of the BFZ. To these ends, the forecast experiments were conducted with a community version of an operational model at a resolution of approximately $20 \mathrm{~km}$.

The remainder of this paper is composed as follows. Section 2 describes the data and experimental design. The results are shown in Section 3. Finally, conclusions and discussion are given in Section 4.

\section{Data and experimental design}

\subsection{Data}

JMA Radar-Automated Meteorological Data Acquisition System (AMeDAS) was used for precipitation analysis. The Japanese 55-year Reanalysis (Kobayashi et al. 2015) was used for the analysis of other atmospheric variables. In addition, the Regional Specialized Meteorological Center (RSMC) Tokyo-Typhoon Center best track (denoted simply as best track hereafter) was used for analysis of the position and pressure of the cyclonic centre.

\subsection{Experimental design}

Forecast experiments were conducted with the European Centre for Medium-Range Weather Forecasts (ECMWF) OpenIFS (Szépszó et al. 2019) Cy40r1v2 (Cycle 40 release 1 version 2), which was operational between November 2013 and May 2015. OpenIFS is a community version of the ECMWF Integrated Forecast System (IFS) without the data assimilation component. The model resolution is TL1023L60 i.e., the truncation wave number is 1023 using triangular truncation on a linear grid, approximately $0.18^{\circ}$ in the horizontal grid, and 60 layers in the vertical grid. The model was initialized with ECMWF operational analysis and integrated up to 12 UTC, 8 July. The initial time was varied between 12 UTC, 28 June, and 12 UTC, 3 July, with an interval of $12 \mathrm{~h}$.

\subsection{Tracking and axisymmetric analysis}

The cyclonic centre in this study is defined by the minimum sea-level pressure. Biquadratic interpolation was used to find the minimum from the values at nine grid points.

In the present study, axisymmetric analysis was conducted by spherical orthodrome transformation (Ritchie 1987; Nakamura et al. 1997; Yamazaki 2011). In the transformed coordinates, the cyclonic centre is relocated to the North Pole. The coordinate transform is easily conducted in three-dimensional Cartesian coordinates (Appendix A).

\section{$2.4 Q$-vector analysis}

To investigate the role of Prapiroon in Baiu frontogenesis, Q-vector analysis (Hoskins et al. 1978) was conducted. The divergence of the Q-vector is regarded as a driver of the vertical motion in the omega equation in pressure coordinates.

$$
\left(S_{0} \nabla^{2}+f_{0}^{2} \frac{\partial^{2}}{\partial p^{2}}\right) \omega=-2 \nabla \cdot \mathbf{Q}
$$

where 

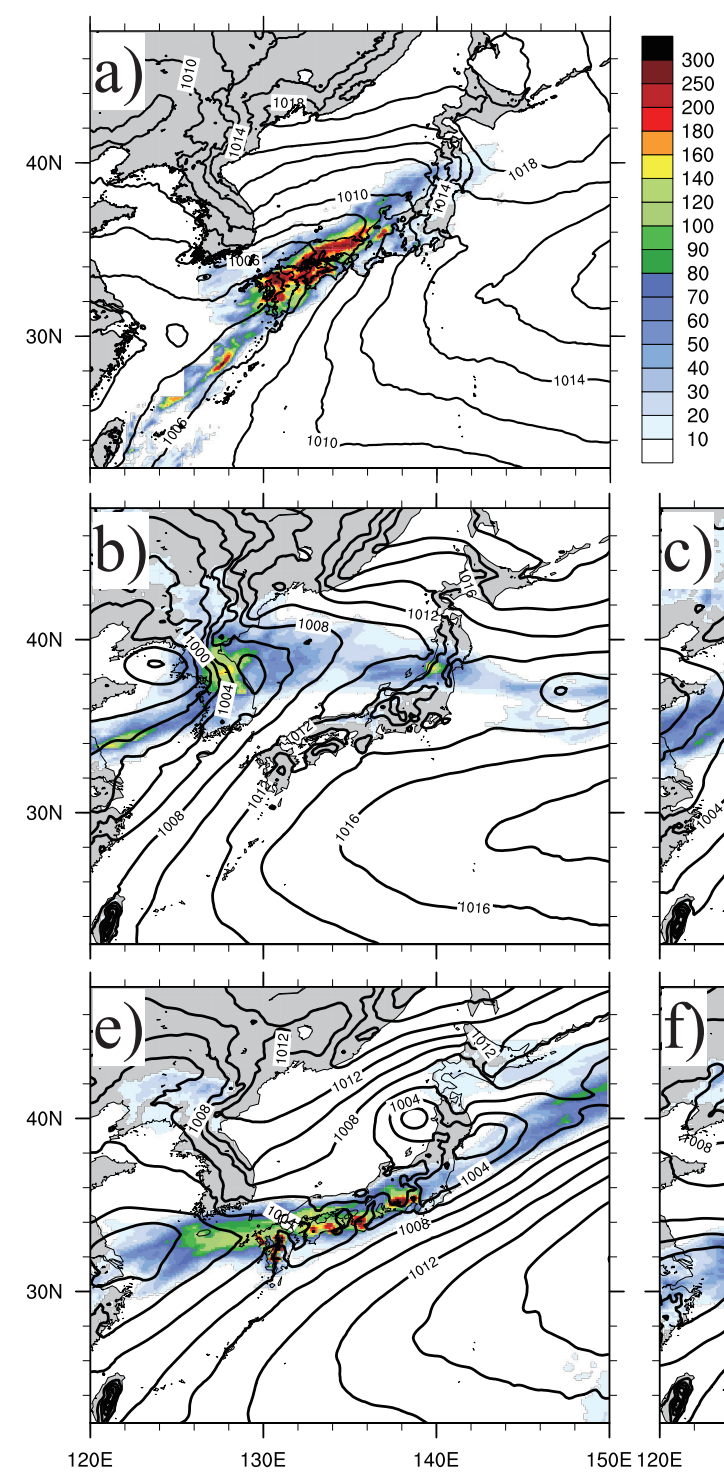
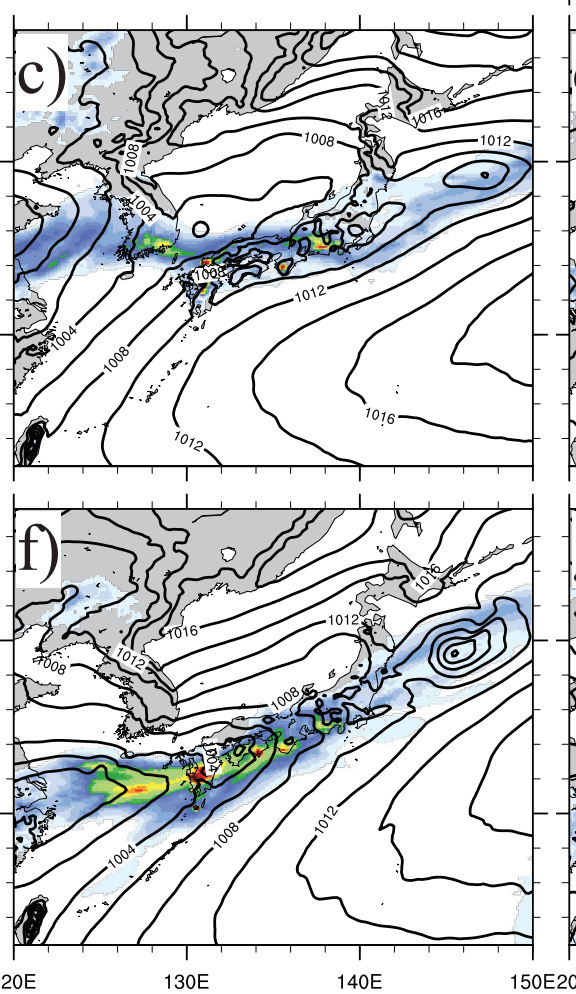

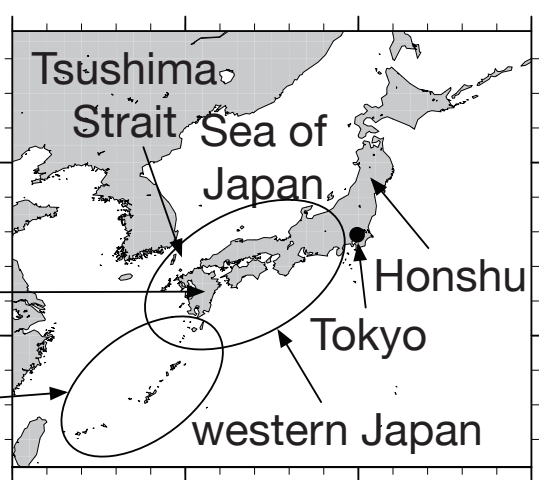

Nansei Islands
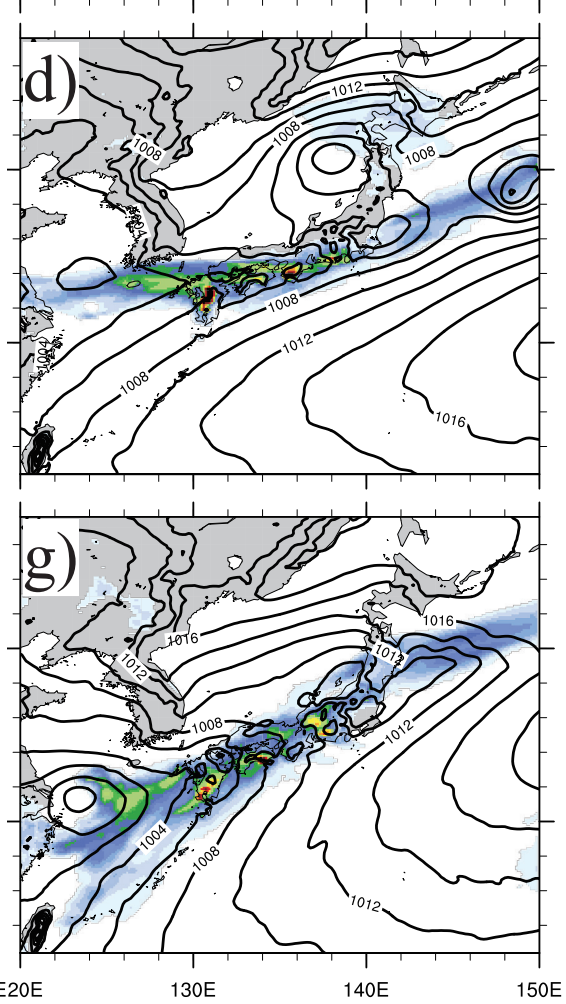

Fig. 1. Daily accumulated precipitation (mm, shades) on 6 July 2018 and sea-level pressure (hPa, contours) at 12 UTC, 6 July, of the a) JMA RadarAMeDAS analysis and of the forecasts initialized at 12 UTC, b) 28, c) 29, d) 30 June, e) 1, f) 2, and g) 3 July. Toponyms are shown in the upper-right inset.

$$
\mathbf{Q}=-\frac{R}{p}\left(\frac{\partial \mathbf{v}_{\mathrm{g}}}{\partial x} \cdot \nabla T, \frac{\partial \mathbf{v}_{\mathrm{g}}}{\partial y} \cdot \nabla T\right)
$$

where $x=a \lambda \cos \phi, y=a \phi, a$ is the Earth's radius, $\lambda$ is the longitude, $\phi$ is the latitude, $R$ is the gas constant for dry air, $p$ is the pressure, $\mathbf{v}_{\mathrm{g}}$ is the geostrophic wind, and $T$ is the temperature (Sanders and Hoskins 1990). In addition, the Q-vector is a measure of the intensity of the temperature gradient, or frontogenesis function, as follows:

$$
\frac{\mathrm{d}_{\mathrm{g}}}{\mathrm{d} t}|\nabla T|^{2}=\left(\frac{\partial}{\partial t}+\mathbf{v}_{\mathrm{g}} \cdot \nabla\right)|\nabla T|^{2}=2 \frac{p}{R} \mathbf{Q} \cdot \nabla T .
$$

Isotropic spatial smoothing in the spectral space was applied to eliminate small-scale noise and to highlight synoptic-scale features. The filter has the form $\exp \left\{[-n(n+1) / N(N+1)]^{r}\right\}$, where $n$ is the total wave number and $N$ is the total wave number at which the amplitude is reduced to $e^{-1}, r$ is a parameter for scale selectivity (Sardeshmukh and Hoskins 1984). Here, $N=62$ for $\nabla \cdot \mathbf{Q}$, $N=84$ for $\mathbf{Q} \cdot \nabla T$, and $r=2$.

\section{Results}

\subsection{Precipitation}

During HRE1807, the rainfall amount was particularly large between 5 and 7 July and peaked on 6 July. Figure 1a shows the JMA Radar-AMeDAS daily precipitation analysis on 6 July. A trough extends northeastward from southeastern China towards the Japanese Isles with the daily accumulated precipitation larger than $100 \mathrm{~mm} \mathrm{~d}^{-1}$ over western Japan between approximately $129^{\circ} \mathrm{E}$ and $138^{\circ} \mathrm{E}$.

In the forecast initialized at 12 UTC, 28 June (Fig. 1b), a much weaker precipitation band is formed over the Korean Peninsula and in the Sea of Japan. In the forecast initialized at 12 UTC, 29 June (Fig. 1c), a zonally elongated precipitation band starts to appear over western Japan, but the overall daily accumulated precipitation is relatively small $\left(<100 \mathrm{~mm} \mathrm{~d}^{-1}\right)$, except for several locations. In addition, the trough is displaced somewhat northward. In the forecast from 12 UTC, 30 June, and onwards (Figs. $1 \mathrm{~d}, 1 \mathrm{e}, 1 \mathrm{f}$, and $1 \mathrm{~g}$ ), an intense precipitation band embedded in the trough over western Japan is simulated, although the precipitation amount is significantly underestimated. 
a)

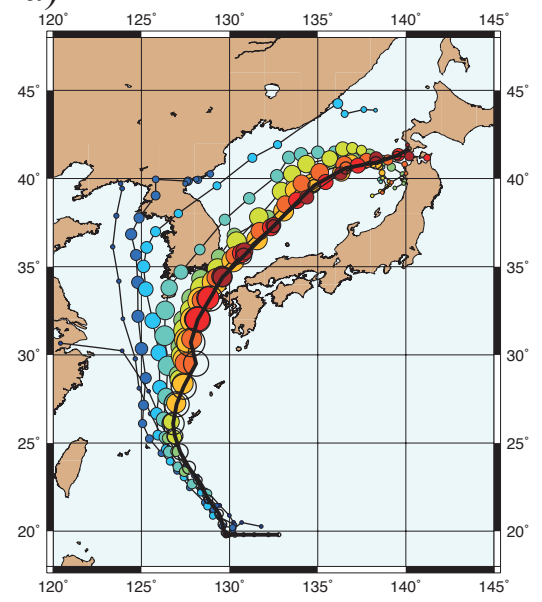

b)

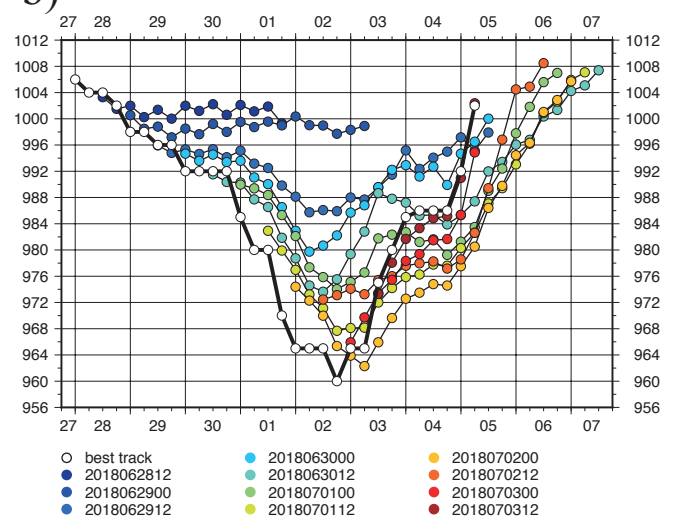

Fig. 2. a) Tracks and b) time evolution of the central pressure (hPa) of the JMA best track (black curves and open circles) and of the forecasts initialized between 12 UTC, 28 June, and 12 UTC, 3 July, every $12 \mathrm{~h}$ (see the legend).

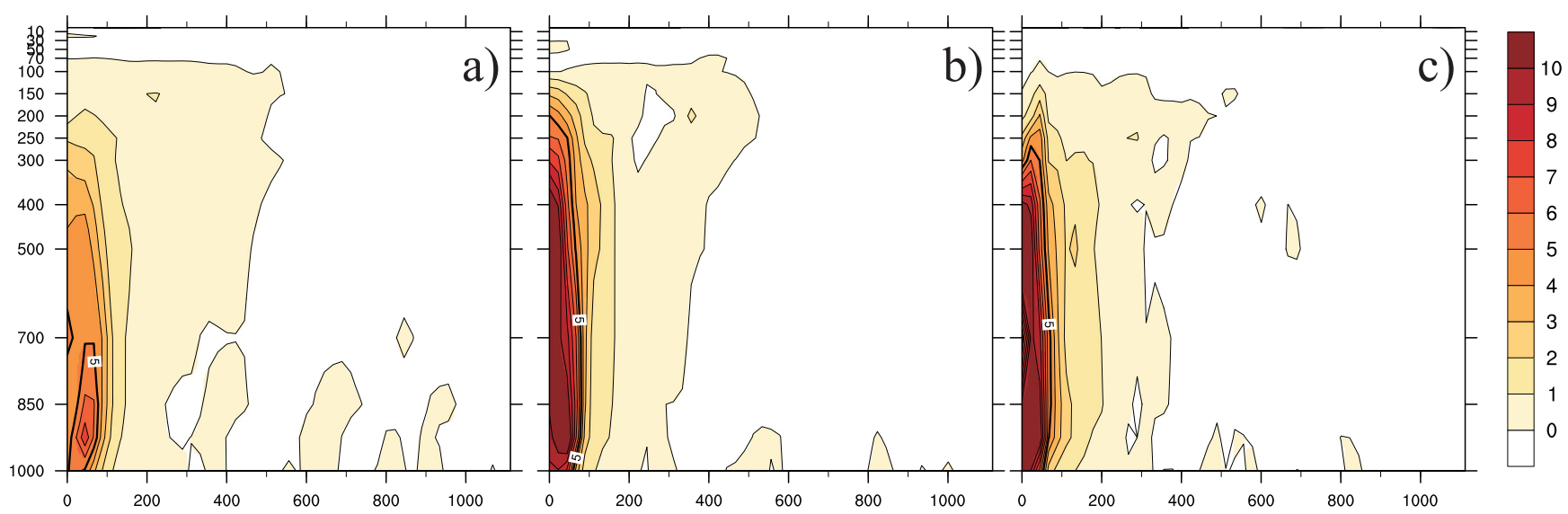

Fig. 3. Cross sections of axisymmetric vorticity $\left(\times 10^{-4} \mathrm{~s}^{-1}\right)$ valid at 12 UTC, 2 July. Forecast initialized at 12 UTC, a) 29 , b) 30 June, and c) ECMWF operational analysis. The contour for $5 \times 10^{-4} \mathrm{~s}^{-1}$ is drawn in a thick curve. The abscissa is the distance from the centre (km), and the ordinate is the pressure.

\subsection{Typhoon tracks and intensity}

Prapiroon passed the Tsushima Strait, migrated northeastward in the Sea of Japan and dissipated near $140^{\circ} \mathrm{E}, 42^{\circ} \mathrm{N}$, according to the best track (Fig. 2a, black curve and open circles). The typhoon tracks in the forecasts initialized at 00 UTC, 1 July, or later converge towards the best track. In contrast, westward bias increases systematically as the lead time lengthens. The typhoon in the forecast initialized at 12 UTC, 30 June, lands in the southern Korean Peninsula and then migrates northeastward in the Sea of Japan to disappear near the location close to that in the best track. The tracks in the forecasts initialized earlier deviate significantly to the west.

It is worthwhile to note that Prapiroon develops further as the track error decreases (Figs. 2a and 2b). In the forecasts from 12 UTC, 28 June, and from 00 UTC, 29 June, with large westward bias, the cyclone hardly develops. In the forecasts from 12 UTC, 30 June, or later, the cyclone migrates northeastward in the Sea of Japan and develops as rapidly as in the best track. The analyzed intensity is not as strong as that in the best track, and the forecasts have some bias in spin up and spin down even with short lead times.

The distributions of predicted precipitation (Figs. 1c and 1d) and typhoon track and intensity (Figs. $2 \mathrm{a}$ and $2 \mathrm{~b}$ ) show a marked jump in predictability between the forecasts from 12 UTC, 29 June, and 12 UTC, 30 June. Thus, comparisons are made between the analysis and these two forecasts in the rest of the paper.
Axisymmetric vorticity analysis (Subsection 2.3 and Appendix A) was conducted to examine the vertical structure. Figure 3 compares the cross sections of vorticity valid at 12 UTC, 2 July, when the typhoon is located near $30^{\circ} \mathrm{N}$ prior to recurvature (Fig. $2 a)$. Note that synoptic conditions between the two forecasts valid 12 UTC, 2 July are quite similar (not shown). In the forecast from 12 UTC, 29 June, the vortex does not develop deeply and is weak (Fig. 3a), while the vortex in the forecast from 12 UTC, 30 June (Fig. 3b), is as deep and strong as that in the analysis (Fig. 3d). The thick contour represents a constant vorticity of $5 \times 10^{-4} \mathrm{~s}^{-1}$ arbitrarily chosen to be the middle of the steep vorticity gradient in the analysis (Fig. 3c). At 12 UTC, 2 July, the vorticity contours of $5 \times 10^{-4} \mathrm{~s}^{-1}$ in the typhoon in the forecast initialized at $12 \mathrm{UTC}$, 29 June, are confined below $700 \mathrm{hPa}$, and those in the forecast initialized at 12 UTC, 30 June, or later extend to approximately $250 \mathrm{hPa}$, as found in the analysis.

Although the precise estimation of the steering flow speed requires further elaboration (Chan 1985; Velden and Leslie 1991), it is reasonable to consider that the typhoon initialized at 12 UTC, 29 June, is advected by the winds averaged in the lower troposphere, and those after are advected by the winds averaged over the entire troposphere. The axially non-symmetric winds (not shown) averaged below $700 \mathrm{hPa}$ are southerly; therefore, the shallow vortex in the unsuccessful forecast continues to migrate northward. In contrast, the axially non-symmetric winds averaged below $300 \mathrm{hPa}$ are southwesterly and act to recurve the deep 

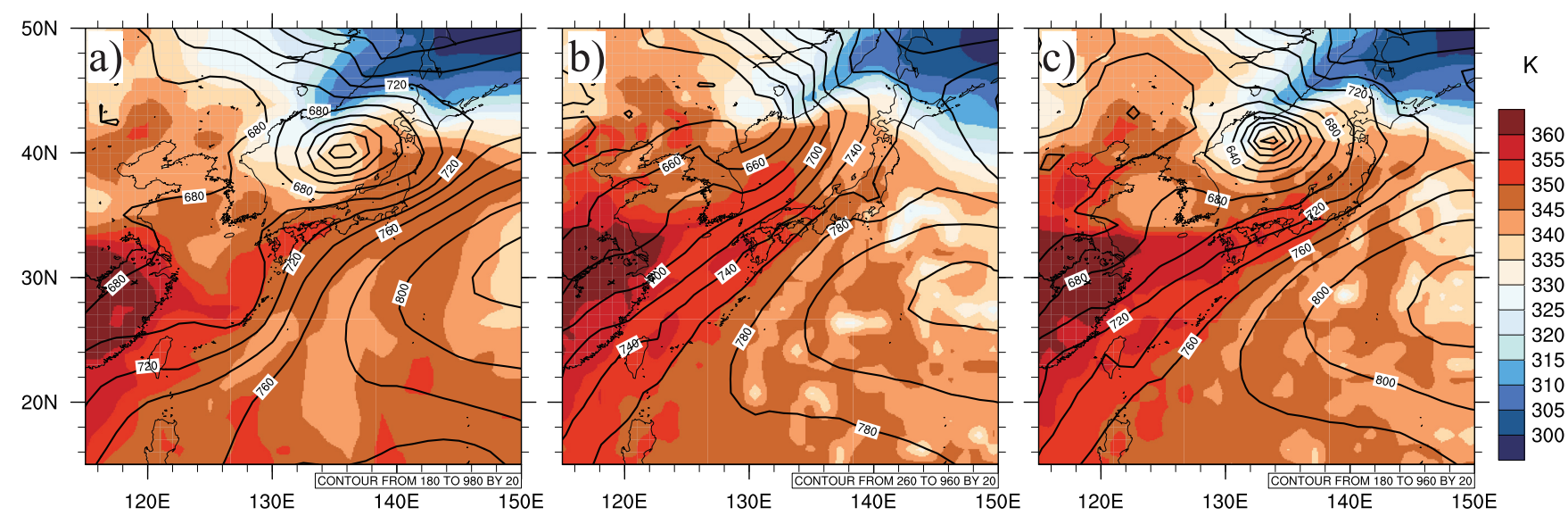

Fig. 4. Equivalent potential temperature (K, shades) and geopotential height (gpm, contours) on the $925 \mathrm{hPa}$ surface of a) JRA-55 and of the forecasts initialized at 12 UTC, b) 29, c) 30 June 2018 valid at 12 UTC, 4 July 2018.

vortex in the successful forecast as in the analysis.

\subsection{Intensification of the $\mathrm{BFZ}$}

Figure 4 compares the equivalent potential temperature $\left(\theta_{\mathrm{e}}\right)$ on the $925 \mathrm{hPa}$ surface at $12 \mathrm{UTC}, 4$ July. The JRA-55 analysis indicates that a warm and moist airmass with $\theta_{\mathrm{e}}>360 \mathrm{~K}$ spread over southeastern China, and a filament with $\theta_{\mathrm{e}}>350 \mathrm{~K}$ extended towards Japan along a belt with a large pressure gradient between Prapiroon and the Pacific anticyclone (Fig. 4a). The high $\theta_{\mathrm{e}}$ air is wound into the tropical cyclone from its southeastern sector. Similar $\theta_{\mathrm{e}}$ distributions are reproduced in the forecasts from 12 UTC, 30 June, and later despite a weak southerly component in the Nansei Islands near $130^{\circ} \mathrm{E}$ and $30^{\circ} \mathrm{N}$ (Fig. 4c). Note that Prapiroon also acts to draw low $\theta_{\mathrm{e}}$ air from the Okhotsk anticyclone into the western Sea of Japan. As a result, the northward spread of warm air is blocked, which enhances the equivalent potential gradient in western Japan (Moteki 2019). This intensification of the BFZ on 4 July can be regarded as the preconditioning stage of HRE1807.

By contrast, in the forecasts from 12 UTC, 29 June (Fig. 4b), and older, the lack of the tropical cyclone allows the Pacific anticyclone to extend northwestward with large pressure gradients in the Sea of Japan. As a result, the high $\theta_{\mathrm{e}}$ air extends northeastward from southeastern China towards the Sea of Japan to bring precipitation over the Korean Peninsula instead of over Japan (Figs. 1b and 1c).

\subsection{Q-vector analysis}

In the mid-latitude baroclinic zone, the temperature gradient is dominantly meridional, i.e., $\nabla T \approx \partial T / \partial y$, and the Q-vector (2) is simplified to

$$
\mathbf{Q}=-\frac{R}{p}\left(\frac{\partial v_{\mathrm{g}}}{\partial x}, \frac{\partial v_{\mathrm{g}}}{\partial y}\right) \frac{\partial T}{\partial y}
$$

i.e., the gradient of the meridional wind determines the Q-vector (Hoskins et al. 1978, Fig. 5). Because $\partial v_{\mathrm{g}} / \partial x$ is negative to the west and positive to the east of the northerly maximum of $v_{\mathrm{g}}$ and $\partial v_{\mathrm{g}} / \partial y$ is positive to the north and negative to the south, the Q-vector is divergent around the northerly maximum of the cyclone embedded in the baroclinic zone.

Figure 6 shows the Q-vectors calculated from the JRA-55 analysis (Fig. 6a) and OpenIFS forecasts (Figs. 6b and 6c) on 5 July. In the analysis (Fig. 6a), a pair of divergence (centred at approximately $138^{\circ} \mathrm{E}, 41^{\circ} \mathrm{N}$ ) and convergence (approximately $142^{\circ} \mathrm{E}, 42^{\circ} \mathrm{N}$ ) in northern Japan corresponds to the northerly near and southerly due to the typhoon. The southward vectors to the south of the divergent region near $128^{\circ} \mathrm{E}-144^{\circ} \mathrm{E}, 35^{\circ} \mathrm{N}-38^{\circ} \mathrm{N}$ are directed against the temperature gradient to indicate frontogenesis (Fig. 6d). These vectors are convergent over northern Kyushu

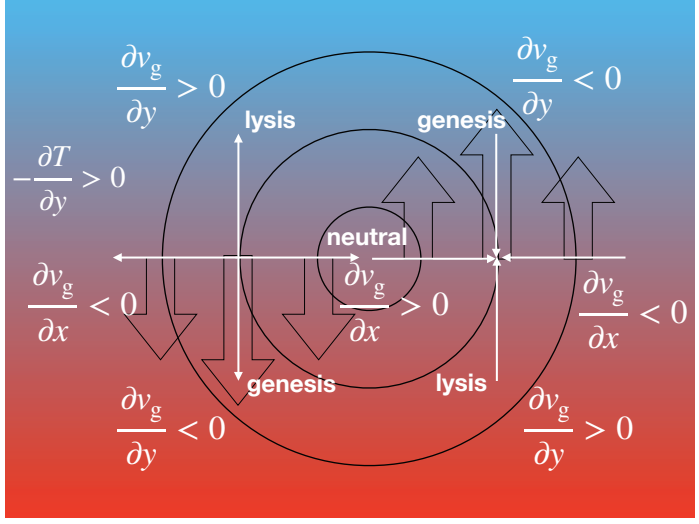

Fig. 5. Schematic diagram showing the directions of Q-vectors (arrows) from a cyclone (contours of geopotential and thick arrows of meridional geostrophic winds) in only the southward temperature gradient (colour shade). See the text for details.

near $128^{\circ} \mathrm{E}, 34^{\circ} \mathrm{N}$, and over central Japan (approximately $132^{\circ} \mathrm{E}-$ $140^{\circ} \mathrm{E}, 34^{\circ} \mathrm{N}-38^{\circ} \mathrm{N}$ ). Note that the Q-vectors that intensify the BFZ emanate from Prapiroon rather than directly from the Okhotsk anticyclone. The forecast from 12 UTC, 29 June, lacks these features (Fig. 6b) with little hint of frontogenesis (Fig. 6e). The forecasts from 12 UTC, 30 June, and later have a divergence-convergence pair similar to that in the analysis. Although the amplitudes are overestimated due to the slow dissipation and the phase is shifted westward due to slow eastward migration of the typhoon (Fig. 2b), the Q-vectors from the divergence associated with the northerly winds are directed southward to indicate the intensification of the temperature gradient (Fig. 6f). The contrast between the Q-vectors of the successful and unsuccessful forecasts indicates that Prapiroon acts to enhance the Baiu frontal zone by intensifying the temperature gradient and inducing ascent in the preconditioning stage on 4 July of HRE1807.

\section{Concluding remarks}

Forecast experiments were conducted to investigate the predictability of the Heavy Rainfall Event in July 2018. The intensification of the Baiu frontal zone and heavy precipitation in western Japan were found to be predicted from 12 UTC, 30 June (a lead time of approximately 5.5 days), and later. The precipitation shifted northward and became weak with longer lead times. 

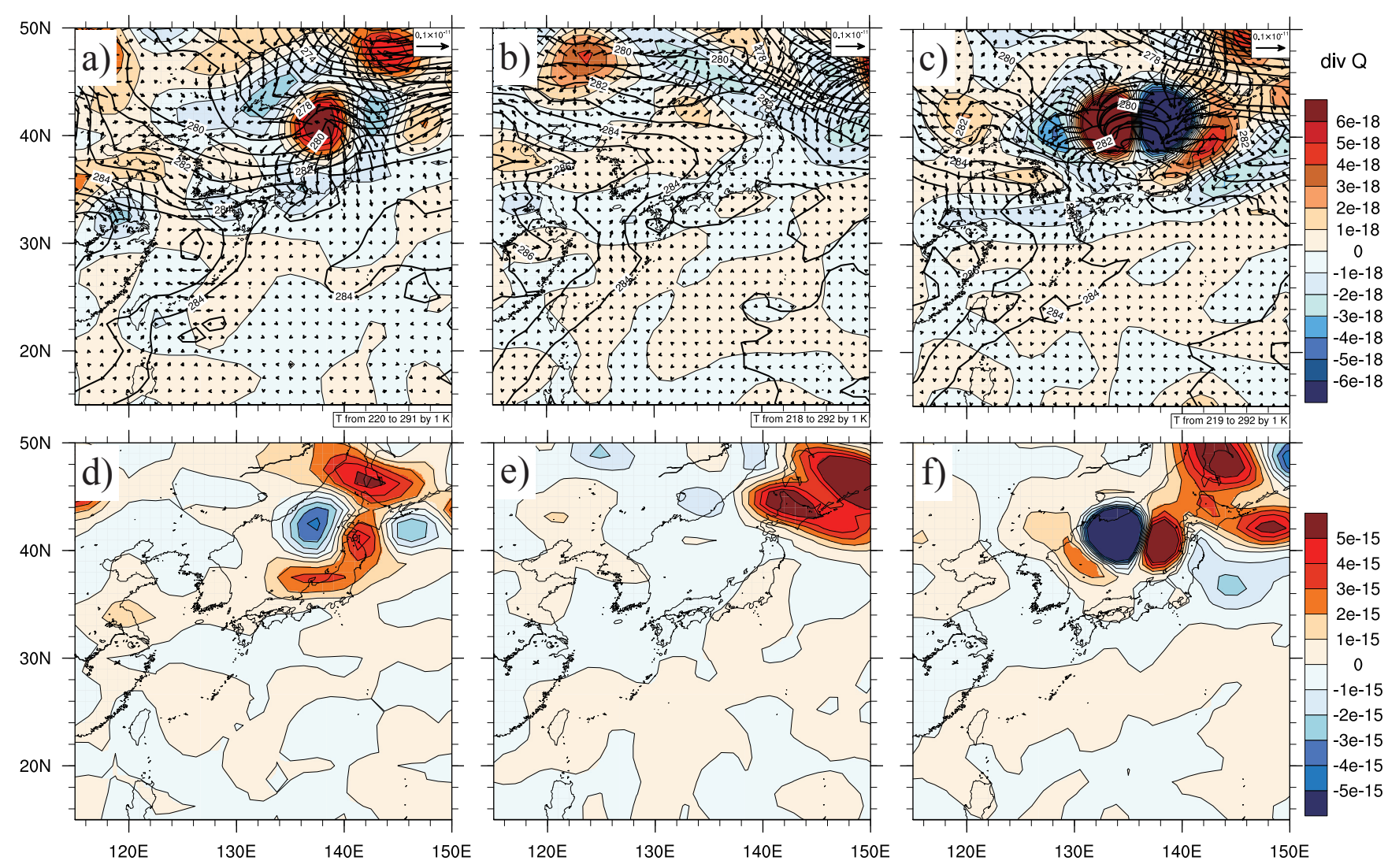

Fig. 6. Temperature (K, contours), Q-vectors $\left(\mathrm{m}^{2} \mathrm{~kg}^{-1} \mathrm{~s}^{-1}\right.$, arrows) and its divergence ( $\mathrm{m} \mathrm{kg}^{-1} \mathrm{~s}^{-1}$, shades) (a-c) and frontogenesis function $\mathrm{d}_{\mathrm{g}}|\Delta T|^{2} / \mathrm{d} t$ $\left(\mathrm{K}^{2} \mathrm{~s}^{-1} \mathrm{~m}^{-2}\right)(\mathrm{d}-\mathrm{f})$ on 5 July 2018. Produced from a, d) JRA-55 and forecasts from $\left.12 \mathrm{UTC}, \mathrm{b}, \mathrm{e}\right)$ 29, c, f) 30 June 2018.

Comparisons with successful and unsuccessful forecasts indicated the role of Typhoon Prapiroon in preconditioning the environment to be suitable for heavy rainfall by drawing warm airmass from the south and cold airmass from the north, consistent with the Moteki (2019)'s analysis. In the successful forecasts, the typhoon travelled northeastward in the Sea of Japan and intensified the BFZ. In the unsuccessful forecasts, the typhoon did not develop to reach the upper troposphere and failed to be advected eastward by the mid-latitude westerlies.

Our experiments with approximately $20-\mathrm{km}$ horizontal resolution, much finer than those used in Kotsuki et al. 2018 and in Matsunobu and Matsueda 2019, were able to simulate the vertical structure of Prapiroon and warm and cold air filaments realistically to demonstrate the link between Prapiroon and HRE1807. Mid-latitude processes, such as the propagation of the Rossby waves along the subtropical jet and associated intensification of the subtropical anticyclone, may reinforce the Baiu frontal zone (Enomoto et al. 2009). An analysis of the JMA weekly forecast indicates that the subtropical jet seems to have a longer predictability than that of the tropical cyclone (Kuwano-Yoshida pers. comm.). Operational ensemble track forecasts are consistent with our deterministic track forecast experiments: prior to 12 UTC, 30 June (Matsunobu and Matsueda 2019). Their results may indicate that initial perturbations could not remedy the growth of the tropical cyclone in this case.

In hindsight, the forecast failed because of underestimation of the intensity of the typhoon. It is implied that the enhanced vortex would improve the track forecast and the subsequent processes leading to the heavy precipitation. The ensemble forecast could also be recentralized with the intensified typhoon (Chang et al. 2014). Such deterministic and ensemble forecasts may have practical benefit for scenario-based warnings.

\section{Appendix A}

Consider a longitude-latitude grid, where the cyclone relocated at the North Pole (the NP coordinates hereafter). The transformation between the original and NP coordinates is straightforward with rotations in Cartesian coordinates. A positional vector in the Cartesian coordinates $\boldsymbol{r}=(x, y, z)^{\mathrm{T}}$ is obtained from the longitudelatitude coordinates by

$$
\begin{aligned}
& x=\cos \lambda \sin \theta \\
& y=\sin \lambda \sin \theta \\
& z=\cos \theta
\end{aligned}
$$

Now, $\boldsymbol{r}$ in the NP coordinates is projected to the original coordinates by following two rotations. Assuming that the cyclonic centre is $\left(\lambda_{\mathrm{c}}, \theta_{\mathrm{c}}\right)$, where $\lambda_{\mathrm{c}}$ and $\theta_{\mathrm{c}}$ are the central longitude and colatitude, respectively:

1. Rotate $-\theta_{\mathrm{c}}$ around the $y$ axis (negative because of a clockwise rotation in the $x-z$ plane).

2 . Rotate $\lambda_{\mathrm{c}}$ around the $z$ axis.

The two rotations are described by the following rotation matrix:

$$
\begin{aligned}
A_{1} & =\left[\begin{array}{ccc}
\cos \theta_{\mathrm{c}} & 0 & \sin \theta_{\mathrm{c}} \\
0 & 1 & 0 \\
-\sin \theta_{\mathrm{c}} & 0 & \cos \theta_{\mathrm{c}}
\end{array}\right] \\
A_{2} & =\left[\begin{array}{ccc}
\cos \lambda_{\mathrm{c}} & -\sin \lambda_{\mathrm{c}} & 0 \\
\sin \lambda_{\mathrm{c}} & \cos \lambda_{\mathrm{c}} & 0 \\
0 & 0 & 1
\end{array}\right] \\
A & =A_{2} A_{1}=\left[\begin{array}{ccc}
\cos \lambda_{\mathrm{c}} \cos \theta_{\mathrm{c}} & -\sin \lambda_{\mathrm{c}} & \cos \lambda_{\mathrm{c}} \sin \theta_{\mathrm{c}} \\
\sin \lambda_{\mathrm{c}} \cos \theta_{\mathrm{c}} & \cos \lambda_{\mathrm{c}} & \sin \lambda_{\mathrm{c}} \sin \theta_{\mathrm{c}} \\
-\sin \theta_{\mathrm{c}} & 0 & \cos \theta_{\mathrm{c}}
\end{array}\right]
\end{aligned}
$$


The points in the NP coordinates are transformed to the original coordinates and are obtained by $A \boldsymbol{r}$. The longitude and latitude corresponding to $A \boldsymbol{r}$ are obtained by

$$
\begin{aligned}
& \lambda= \begin{cases}\operatorname{atan} \frac{y}{x} \bmod 2 \pi, & x \neq 0 \\
0, & x=0\end{cases} \\
& \theta=\operatorname{acos} z .
\end{aligned}
$$

The scalar field is interpolated at $(\lambda, \theta)$. The zonally symmetric and asymmetric components in the transformed coordinates represent the axially symmetric and asymmetric components, respectively.

The vectors such as winds need additional procedures. First, the horizontal winds expressed in the Cartesian coordinates are

$$
\begin{aligned}
& \dot{x}=-u \sin \lambda-v \cos \lambda \cos \theta \\
& \dot{y}=u \cos \lambda-v \sin \lambda \cos \theta \\
& \dot{z}=v \sin \theta
\end{aligned}
$$

and each component of a vector in the Cartesian coordinates (A4) is interpolated as a scalar. Then, the coordinates are rotated with $A^{\mathrm{T}} \dot{\boldsymbol{x}}$, where $A^{\mathrm{T}}=A_{1} A_{2}$, to obtain winds in the NP coordinates. The winds in the longitude-latitude coordinates are obtained from those in the Cartesian coordinates by

$$
\begin{aligned}
& u=\dot{y} \cos \lambda-\dot{x} \sin \lambda \\
& v=\operatorname{sgn}(\dot{z}) \sqrt{(\dot{x} \cos \lambda+\dot{y} \sin \lambda)^{2}+\dot{z}^{2}}
\end{aligned}
$$

The transformed zonal and meridional winds represent tangential and radial components, respectively. In this study, the grid spacing is uniform in longitude $(\lambda)$ and in colatitude $(\theta=\pi / 2-\phi)$, and the meridional extent is $10^{\circ}$ from the North Pole.

\section{Acknowledgements}

The present work was supported by JSPS KAKENHI Grant Number JP26282111 and 19H05698. ECMWF OpenIFS was used on the supercomputer system of Kyoto University under a collaboration with ECMWF. ECMWF analysis was provided by Dr. Glen Carver, ECMWF. JMA Radar-AMeDAS and JRA-55 datasets were obtained from the Global Atmospheric Observation Database of the Research Institute for Sustainable Humanosphere, Kyoto University. A portion of the JMA data used in this study was provided by the Meteorological Research Consortium, a framework for research cooperation of the JMA and the Meteorological Society of Japan.

Edited by: S.-K. Park

\section{References}

Chan, J. C. L., 1985: Identification of the steering flow for tropical cyclone motion from objectively analyzed wind fields. Mon. Wea. Rev., 113, 106-116, doi:10.1175/1520-0493(1985)113 $<0106$ :IOTSFF $>2.0 . \mathrm{CO} ; 2$.

Chang, C.-C., S.-C. Yang, and C. Keppenne, 2014: Applications of the mean recentering scheme to improve typhoon track prediction: A case study of Typhoon Nanmadol (2011). J. Meteor. Soc. Japan, 92, 559-584, doi:10.2151/jmsj.2014604.

Enomoto, T., H. Endo, Y. Harada, and W. Ohfuchi, 2009: Relationship between high-impact weather events in Japan and propagation of Rossby waves along the Asian Jet in July 2004. J. Meteor. Soc. Japan, 87, 139-156, doi:10.2151/jmsj. 87.139.

Hoskins, B. J., I. Draghici, and H. C. Davies, 1978: A new look at the $\omega$-equation. Quart. J. Roy. Meteor. Soc., 104, 31-38, doi:10.1002/qj.49710443903.

Kobayashi, S., and co-authors, 2015: The JRA-55 Reanalysis: General specifications and basic characteristics. J. Meteor. Soc. Japan, 93, 5-48, doi:10.2151/jmsj.2015-001.

Kotsuki, S., K. Terasaki, K. Kanemaru, M. Satoh, T. Kubota, and T. Miyoshi, 2019: Predictability of record-breaking rainfall in Japan in July 2018: Ensemble forecast experiments with the near-real-time global atmospheric data assimilation system NEXRA. SOLA, 15A, 1-7, doi:10.2151/sola.15A-001.

Matsunobu, T., and M. Matsueda, 2019: Assessing the predictability of heavy rainfall events in Japan in early July 2018 on medium-range timescales. SOLA, 15A, 19-24, doi:10.2151/ sola.15A-004.

Moteki, Q., 2019: Role of Typhoon Prapiroon (Typhoon No. 7) on the formation process of the Baiu front inducing heavy rain in July 2018 in western Japan. SOLA, 15A, 37-42, doi: 10.2151/sola.15A-007.

Nakamura, H., M. Nakamura, and J. L. Anderson, 1997: The role of high- and low-frequency dynamics in blocking formation. Mon. Wea. Rev., 125, 2074-2093, doi:10.1175/1520-0493 (1997) 125<2074:TROHAL>2.0.CO;2.

Ritchie, H., 1987: Semi-lagrangian advection on a Gaussian grid. Mon. Wea. Rev., 115, 608-619.

Sanders, F., and B. J. Hoskins, 1990: An easy method for estimation of Q-vectors from weather maps. Wea. Forecasting, 5, 346-353, doi:10.1175/1520-0434(1990)005<0346: AEMFEO $>2.0 . \mathrm{CO} ; 2$.

Sardeshmukh, P. D., and B. I. Hoskins, 1984: Spatial smoothing on the sphere. Mon. Wea. Rev., 112, 2524-2529, doi:10.1175/ 1520-0493(1984)112<2524:SSOTS >2.0.CO;2.

Sekizawa, S., T. Miyasaka, H. Nakamura, A. Shimpo, K. Takemura, and S. Maeda, 2019: Anomalous moisture transport and oceanic evaporation during a torrential rainfall event over western Japan in early July 2018. SOLA, 15A, 25-30, doi:10.2151/sola.15A-005.

Shimpo, A., and co-authors, 2019: Primary factors behind the heavy rain event of July 2018 and the subsequent heat wave in Japan. SOLA, 15A, 13-18, doi:10.2151/sola.15A-003.

Szépszó, G., V. Sinclair, and G. Carver, 2019: Using the ECMWF OpenIFS model and state-of-the-art training techniques in meteorological education. 18th EMS Annual Meeting: European Conf. for Applied Meteorology and Climatology 2018, Vol. 16, Advances in Science and Research, Copernicus GmbH, 39-47.

Takemi, T., and T. Unuma, 2019: Diagnosing environmental properties of the July 2018 Heavy Rainfall Event in Japan. SOLA, 15A, 60-65, doi:10.2151/sola.15A-011.

Takemura, K., S. Wakamatsu, H. Togawa, A. Shimpo, C. Kobayashi, S. Maeda, and H. Nakamura, 2019: Extreme moisture flux convergence over western Japan during the Heavy Rain Event of July 2018. SOLA, 15A, 49-54, doi:10.2151/ sola.15A-009.

Tsuguti, H., N. Seino, H. Kawase, Y. Imada, T. Nakaegawa, and I. Takayabu, 2018: Meteorological overview and mesoscale characteristics of heavy rain event of July 2018 in Japan. Landslides, doi:10.1007/s10346-018-1098-6.

Velden, C. S., and L. M. Leslie, 1991: The basic relationship between tropical cyclone intensity and the depth of the environmental steering layer in the Australian region. Wea. Forecasting, 6, 244-253, doi:10.1175/1520-0434(1991)006 $<0244$ :TBRBTC $>2.0 . \mathrm{CO} ; 2$.

Yamazaki, A., 2011: The maintenance mechanism of atmospheric blocking. D.S. thesis, Kyushu University (Available online at http://hdl.handle.net/2324/21709, accessed 25 December 2018).

Manuscript received 11 September 2019, accepted 15 October 2019 SOLA: https://www.jstage.jst.go.jp/browse/sola/ 